\title{
Study of BER in DVB-S2 satellite implemented in Matlab
}

\begin{abstract}
Satellite broadcasting has been considered lately as a promising media for IP streaming due to their wide coverage and high reliable bandwidth. Long propagation delay in GEO satellite system will affect the adaptive algorithms in DVBS-2/RCS systems. Long feedback delay will cause improper physical layer selection of modulation and coding (ModCod). This paper aims to provide an algorithm for physical layer selector in DVB-S2/RCS satellite systems. This algorithm will makeless ModCod switching of the adaptive coding and modulation (ACM) using reduced number of ModCod combinations. We conclude that the number of ModCods in ACM algorithm can be relatively adjusted to maximize DVB-S2 system stability.
\end{abstract}

Keyword: ACM; Physical layer; DVB-S2/RCS; GEO satellite 